\title{
Assessment of a Central Difference Finite Volume Scheme for Modeling of Cavitating Flows Using Preconditioned Multiphase Euler Equations
}

\author{
Kazem Hejranfar \\ Aerospace Engineering Department, \\ Sharif University of Technology, Tehran, Iran \\ khejran@sharif.edu
}

\author{
Kasra Fattah Hesary \\ Aerospace Engineering Department, \\ Sharif University of Technology, Tehran, Iran \\ kasra.fattah@gmail.com
}

\begin{abstract}
A numerical treatment for the prediction of cavitating flows is presented and assessed. The algorithm uses the preconditioned, homogenous, multiphase Euler equations with appropriate mass transfer terms. A cell-centered finite-volume scheme employing the suitable dissipation terms to account for density jumps across the cavity interface is shown to yield an effective method for solving the multiphase Euler equations. The Euler equations are utilized herein for the cavitation modeling, because some certain characteristics of cavitating flows can be obtained using the solution of this system with relative low computational effort. In addition, the Euler equations are appropriate for the assessment of the numerical method used, because of the sensitivity of the solution to the numerical instabilities. For this reason, a sensitivity study is conducted to evaluate the effects of various parameters such as numerical dissipation coefficients and grid size on the accuracy and performance of the solution. The computations are presented for cavitating flows around the NACA0012 and NACA66(MOD) hydrofoils and also an axisymmetric hemispherical fore-body for different conditions and the results are compared with the available numerical and experimental data. The solution procedure presented is shown to be accurate and efficient for predicting different types of cavitating flows over 2D/axisymmetric geometries.
\end{abstract}

\section{INTRODUCTION}

The inception of cavitation occurs due to the drop in pressure of the flow below the vapor pressure of the liquid. The phenomenon is often encountered in a wide variety of liquid handling turbomachineries and devices. Ship propellers, hydraulic turbine and pumps, nozzles and hydrofoils are some examples subject to cavitation. The cavitation phenomenon has been the subject of intensive research because of the effects that it has on performance; undesirable features of cavitation are structural damage, noise and power loss. On the other hand, cavitation is sometimes found useful as its applications in ultrasonic cleaning, enhancement of chemical reactions, biomedical treatments and viscous drag reduction on high speed underwater bodies surrounded fully or partially with a natural or gas-ventilated cavity $[1,2]$. Numerical simulation of cavitating flow is a complicated task, since one has to deal with localized large variations of density which are present within a predominantly incompressible liquid medium, interactions between phases, turbulence, irregularly shaped interfaces and the stiffness in the numerical model. Because of these complexities there is not a single sophisticated mathematical model that can take care of all these factors.

Numerical attempts in the simulation of cavitating flows could be classified broadly under two categories: namely, interface tracking and interface capturing procedures. Interface tracking methods benefit from the assumption of taking the cavitating region at constant pressure equal to the local vapor pressure. Thus, the liquid/vapor interface is treated as part of the boundary and the computations are done for liquid phase only. The interface is updated iteratively until a convergent shape is achieved with a constant pressure along it. Numerically, however, the interface tracking approach has to employ a wake closure model to approximate the two-phase behavior in the wake region at the end of the cavitation region, since it is impossible to impose a constant pressure condition on the entire cavity surface while the recovery of pressure occurs at the aft end of the cavitation [3, 4]. This category is represented by the potential flow based models [5-11] and the Euler and Navier-Stokes equations based models $[4,12,13]$. Adaptations of potential flow methods remain in widespread use today, due to their inherent computational efficiency, and their proven effectiveness in predicting numerous first-order dynamics of supercavitating configurations. However, the use of Euler/Navier-Stokes methods appears to have the advantages over potential flow methods in that the application in complex geometries is not difficult, a priori knowledge of cavity detachment point and bubble length are not required and they do not have the potential flow problems in the presence of rotational effects such as those that arise from non-uniform inflows [13]. Although interface tracking models are able to predict certain characteristics of cavitating flows such as cavity detachment point and pressure distribution, they are only limited to model the stable sheet cavitation, no cavitation is involved, wake model is not physical and it is limited to 2D computation because of the difficulty to track 3D phase interface [14]. 
In the second category, interface capturing methods, an Eulerian approach is used in which each phase is described by separate equations. The most attractive feature of this procedure is that no wake closure model is required and the whole cavity region shape is obtained as a part of the solution. Formulations in this category can be divided in two main groups, namely multi-fluid modeling and mixture modeling, based on the governing equations being used. In the former group, a set of conservation equations is employed for each phase and the interaction terms at the interfaces are added to the equations as source terms [15]. In the second group, mixture modeling, a single fluid is employed for both liquid and vapor in which density varies widely between liquid and vapor extremes. The key differences here mostly come from the relation that defines the variable density field. In one class of methods, a single continuity equation is considered with the density varying between liquid and vapor densities through an equation of state [16-19]. However, most generalized cavitation models have used solving a separate continuity equation for each constituent to account for the separate behavior of each phase. These models are managed either by introducing the mass transfer between vapor and liquid as the source terms $[20,21]$, or by the evolution of a cluster of bubbles according to a simplified Eulerian form of the Reyleigh-Plesset equation [22-24]. Such multi-species methods are also termed homogeneous because there is assumed to be no-slip between constituents in each cell. Models with this strategy can, in principle, cover all types of cavitation.

Since cavitating flows are highly dynamic and typically turbulent, most cavitation simulations deal with the computation of turbulent cavitating flows and therefore NavierStokes equations based models for simulating the cavitation include a turbulent model (for example see Refs. [16,18,20,2527] and others). The most turbulence-dominated region is the wake region at the rear portion of the cavitation that recirculation and wall jet-like flow structure are seen there. Note that the mechanisms of this kind of two-phase flows in the wake region are extremely complex and no available turbulence model is capable of treating this kind of flow properly [22]. Taking into consideration all the issues with turbulence modeling and the benefits from the fact that the Euler equations can provide valuable information about many fundamental phenomena with lower computational effort than the NavierStokes equations, the inviscid calculation of cavitating flows using the preconditioned multiphase Euler equations is pursued in this paper. Although the modeling difficulty of the highly turbulence-dominated two-phase flow region is avoided here and the inviscid computation of cavitation seems to be a simplified case, lack of the damping effect of viscosity makes the computation of cavitating flows using the Euler equations to be a very difficult task; because of high sensitivity of the numerical solution for capturing the cavity interface.

The main objective of the present study is to assess a numerical treatment for computing cavitating flows using the preconditioned multiphase Euler equations. Herein, a cellcentered finite-volume scheme in conjunction with the modified Jameson's type dissipation terms to account for density jumps across the cavity interface is employed to solve the multiphase Euler equations. The Euler equations are used for the cavitation simulation, because some certain characteristics of cavitating flows can be obtained using the solution of this system with relative low computational effort. In addition, the Euler equations are suitable for the assessment of the numerical method implemented, because of the sensitivity of the solution to the numerical instabilities. The cavitation simulations for the NACA0012 and NACA66(MOD) hydrofoils and also an axisymmetric hemispherical fore-body for different conditions are presented. The effects of the numerical dissipation terms and grid size on the results are also investigated and the overall capability and performance of the present modeling are assessed.

\section{GOVERNING EQUATIONS}

For numerical simulation of cavitating flows, an interface capturing method using a mixture model is adopted. The flow is assumed to be in thermal and dynamic equilibrium at the interface where the flow velocity is assumed to be continuous. The equations are described for a fluid mixture of liquid and vapor. The momentum equations are used to describe the fluid mixture motion while a single continuity equation can be used for each fluid component. Herein, the vapor continuity equation is replaced by the mixture continuity equation. In the development of the above system of equations, a mixture volume continuity equation is employed rather than a mixture mass equation. Because of this choice, neither a physical time derivative nor the mixture density appears in the continuity equation, although the mixture density can vary in space and time. To regulate the mass-transfer rate from liquid to vapor $\left(\dot{m}^{-}\right.$) and from vapor to liquid ( $\dot{m}^{+}$), appropriate source terms are added to the mixture and liquid continuity equations. Hence, the preconditioned multiphase Euler equations can be written in the Cartesian form as:

$$
\begin{gathered}
\frac{1}{\rho_{m} \beta^{2}} \frac{\partial p}{\partial \tau}+\frac{\partial u}{\partial x}+\frac{\partial v}{\partial y}+\kappa \frac{v}{y}=\left(\dot{m}^{+}+\dot{m}^{-}\right)\left(\frac{1}{\rho_{l}}-\frac{1}{\rho_{v}}\right) \\
\frac{\partial}{\partial \tau}\left(\rho_{m} u\right)+\frac{\partial}{\partial x}\left(\rho_{m} u^{2}+p\right)+\frac{\partial}{\partial y}\left(\rho_{m} u v\right)+ \\
\kappa \frac{\left(\rho_{m} u v\right)}{y}=0 \\
\frac{\partial}{\partial \tau}\left(\rho_{m} v\right)+\frac{\partial}{\partial x}\left(\rho_{m} u v\right)+\frac{\partial}{\partial y}\left(\rho_{m} v^{2}+p\right)+ \\
\kappa \frac{\left(\rho_{m} v^{2}\right)}{y}=0 \\
\left(\frac{\alpha_{\ell}}{\rho_{m} \beta^{2}}\right) \frac{\partial p}{\partial \tau}+\frac{\partial \alpha_{\ell}}{\partial \tau}+\frac{\partial \alpha_{\ell} u}{\partial x}+\frac{\partial \alpha_{\ell} v}{\partial y}+\kappa \frac{\left(\alpha_{\ell} v\right)}{y}= \\
\frac{1}{\rho_{l}}\left(\dot{m}^{+}+\dot{m}^{-}\right)
\end{gathered}
$$

where $\beta$ is the pseudo-compressibility coefficient or the preconditioning parameter and $\kappa=0$ and 1 denotes $2 \mathrm{D}$ and axisymmetric body, respectively. To render the system hyperbolic and to facilitate the use of time-marching procedures, a pseudo-time derivative term (represented by $\tau$ ) is introduced in the mixture continuity equation; a strategy that 
derives from the work of Chorin [28]. The corresponding artificial time-derivative term is also added to the phasic continuity equation to ensure that the proper differential equation in the non-conservative form is satisfied [20].

The preceding equations (1) are nondimensionalized by the reference parameters. The density is normalized with the liquid density, $\rho_{l}$, the pressure by the dynamic pressure $\left(\rho_{l} U_{\infty}^{2}\right)$., the velocities by the freestream velocity, $U_{\infty}$, and lengths by the characteristic length of the body, $L$. The system of equations (1) can be written in dimensionless and vector form as follows:

$\Gamma \frac{\partial Q}{\partial \tau}+\frac{\partial E}{\partial x}+\frac{\partial F}{\partial y}+\kappa\left(\frac{F+G}{y}\right)-S=0$

where the vector of dependent variables $Q$, the inviscid flux vectors $E, F, G$, and the source terms vector $S$ are given by

$Q=\left(p, u, v, \alpha_{l}\right)^{T}$

$E=\left(u, \rho_{m} u^{2}+p, u v, \alpha_{l} u\right)^{T}$

$F=\left(v, u, \rho_{m} v^{2}+p, \alpha_{l} v\right)^{T}$

$G=(0,0,-p, 0)^{T}$

$S=\left(\left(\dot{m}^{+}+\dot{m}^{-}\right)\left(1-\frac{1}{\rho_{v}}\right), 0,0,\left(\dot{m}^{+}+\dot{m}^{-}\right)\right)^{T}$

and the preconditioning matrix, $\Gamma$, is given by

$$
\Gamma=\left[\begin{array}{cccc}
\frac{1}{\rho_{m} \beta^{2}} & 0 & 0 & 0 \\
0 & \rho_{m} & 0 & u \Delta \rho_{l} \\
0 & 0 & \rho_{m} & v \Delta \rho_{l} \\
\frac{\alpha_{l}}{\rho_{m} \beta^{2}} & 0 & 0 & 1
\end{array}\right]
$$

where $\Delta \rho_{l}$ is the difference between liquid and vapor densities. The normalized mixture density, $\rho_{m}$, and $\Delta \rho_{l}$ are defined by

$\rho_{m}=\alpha_{l}+\alpha_{v} \rho_{v}$

$\Delta \rho_{l}=1-\rho_{v}$

where $\alpha_{l}$ and $\alpha_{v}$ are the volume fractions for the liquid and vapor phases, respectively.

Introducing the total inviscid flux vector as $\vec{H}(\vec{Q})=F(\vec{Q}) \hat{i}+G(\vec{Q}) \hat{j}$, the integral form of preconditioned multiphase Euler equations (2) using the Gauss divergence theorem for an arbitrary control volume $\Omega$ is written as

$$
\frac{d}{d \tau} \iint_{\Omega} \Gamma \vec{Q} d \Omega+\oint_{\partial \Omega} \vec{H} \cdot \hat{n} d l+\kappa \iint_{\Omega}\left(\frac{\vec{F}+\vec{G}}{y}\right) d \Omega=
$$

where $\hat{\boldsymbol{n}}\left(\hat{n}_{x}, \hat{n}_{y}\right)$ is the local outward unit vector normal to the boundary surface, $\vec{V} \cdot \hat{n}=v n_{x}+u n_{y}$ is the normal velocity component of fluid and $\vec{H}_{n}=\vec{H}(Q) \cdot \hat{n}$ is the total inviscid flux normal to the surface of the control volume as

$$
\vec{H}_{n}=\vec{H}(Q) \cdot \hat{n}=(\vec{V} \cdot \hat{n})\left[\begin{array}{c}
1 \\
u \\
v \\
\alpha
\end{array}\right]+p\left[\begin{array}{c}
0 \\
n_{x} \\
n_{y} \\
0
\end{array}\right]
$$

Equation (7) describes a relationship in which the time rate of change of the state vector $Q$ within the domain $\Omega$ is balanced by the net inviscid fluxes across the boundary surface $\partial \Omega$.

\section{CAVITATION MODEL}

To model cavitation, the calculation of evaporation $\dot{m}^{+}$ and condensation $\dot{m}^{-}$is required. The cavitation models used in this study are similar to those used by Kunz et al. [20] and Merkle et. al. [21].

Model of Merkle et al. The transformation of liquid to vapor, $\dot{m}^{-}$, is modeled as being proportional to the liquid volume fraction and the amount by which the pressure is below the vapor pressure. Conversely, $\dot{m}^{+}$is the rate for reconversion of vapor back to liquid in regions where the pressure exceeds the vapor pressure. The normalized conversion rates are specified as follows:

$$
\begin{aligned}
& \dot{m}^{-}=\left(\frac{C_{\text {evap }}}{t_{\infty}}\right) \frac{1}{\rho_{v}} \alpha_{l} \operatorname{Min}\left(0, p-p_{v}\right) \\
& \dot{m}^{+}=\left(\frac{C_{\text {dest }}}{t_{\infty}}\right)\left(1-\alpha_{l}\right) \operatorname{Max}\left(0, p-p_{v}\right)
\end{aligned}
$$

Model of Kunz et al. The transformation of liquid to vapor, $\dot{m}^{-}$, is similar to that used by Merkle et al. [21]. For transformation of vapor to liquid, $\dot{m}^{+}$, a simplified form of Ginzburg-Landau potential is used which is a function of volume fraction. The normalized mass transfer rates are given by following expressions as

$$
\begin{aligned}
& \dot{m}^{-}=\left(\frac{C_{\text {evap }}}{t_{\infty}}\right) \frac{1}{\rho_{v}} \alpha_{l} \operatorname{Min}\left(0, p-p_{v}\right) \\
& \dot{m}^{+}=\left(\frac{C_{\text {dest }}}{t_{\infty}}\right) \rho_{v}\left(1-\alpha_{l}\right) \alpha_{l}^{2}
\end{aligned}
$$

In the both models, $p_{v}$ is the normalized vapor pressure, which is defined in terms of the cavitation number $\sigma$ as follows 
$\sigma=2\left(p_{\infty}-p_{v}\right)$

Both $\frac{C_{\text {evap }}}{t_{\infty}}$ and $\frac{C_{\text {dest }}}{t_{\infty}}$ are empirical constants, which express the time rate of the evaporation and condensation processes with respect to the mean flow time scale $\left(L / U_{\infty}\right)$ [27].

\section{NUMERICAL PROCEDURE}

The numerical solution of the preconditioned multiphase Euler equations (7) is given herein. A cell-centered finite volume method is used to solve these equations. The domain is divided into a finite number of structured quadrilateral cells and Eq. (7) is applied to each cell.

Spatial Discritization. In a cell-centered scheme, the flow quantities are associated with the center of each control volume. By discretizing the spatial terms, a system of firstorder ODE is obtained. Using the mean value theorem, Eq. (7) can be written in the following semi-discrete form for each cell

$\Omega_{i, j} \frac{d \vec{Q}_{i, j}}{d \tau}=-\vec{R}\left(\vec{Q}_{i, j}\right)$

where $\Omega_{i, j}$ is the cell area and $\vec{R}\left(\vec{Q}_{i, j}\right)$ is the residual vector defined as

$$
\begin{aligned}
\vec{R}\left(\vec{Q}_{i, j}\right) & =\Gamma_{i, j}^{-1}\left(\sum_{n c f=1}^{4}\left[\vec{H}_{n} \Delta l\right]_{n c f}+\right. \\
& \left.\kappa \frac{\vec{F}\left(\vec{Q}_{i, j}\right)+\vec{G}\left(\vec{Q}_{i, j}\right)}{y_{i, j}} \Omega_{i, j}-\vec{S}_{i, j} \Omega_{i, j}\right)
\end{aligned}
$$

Here, $i$ and $j$ locate a particular cell, $\Delta l$ is the length of each face and summation is performed over all $n c f$ faces of the cell and $\Gamma^{-1}$ denotes the inverse of the preconditioning matrix. The values of the total flux $\vec{H}_{n}(Q)$ on each face of a cell can be evaluated by averaging from its values at the two control points located on the opposite sides of the cell interface, i.e.,

$$
\vec{H}_{n}\left(Q_{i+1 / 2, j}\right)=\frac{1}{2}\left[\vec{H}\left(Q_{i, j}\right)+\vec{H}\left(Q_{i+1, j}\right)\right]
$$

In this way, the scheme reduces to a second-order central difference on a uniform Cartesian grid.

Artificial Dissipation Terms. Owing to the central difference nature of the scheme, stabilization is required to eliminate spurious oscillations. Scalar-valued dissipation, as initially proposed by Jameson et al. [29] is implemented to eliminate odd-even decoupling of the solution and damp oscillations near sudden changes in flow properties. The artificial dissipation term $\vec{D}$ is added to Eq. (14) as follows

$$
\begin{aligned}
\vec{R}\left(\vec{Q}_{i, j}\right) & =\Gamma_{i, j}^{-1}\left(\sum_{n c f=1}^{4}\left[\vec{H}\left(\vec{Q}_{i, j}\right) . \hat{n} \Delta l\right]_{n c f}+\right. \\
& \left.\kappa \frac{F\left(\vec{Q}_{i, j}\right)+G\left(\vec{Q}_{i, j}\right)}{y_{i, j}} \Omega_{i, j}-\vec{S}_{i, j} \Omega_{i, j}-\vec{D}\left(\vec{Q}_{i, j}\right)\right)
\end{aligned}
$$

where the net dissipation flux, $\vec{D}\left(\vec{Q}_{i, j}\right)$ for the cell $(i, j)$ is a blend of second and fourth differences with the coefficients which depend on the local gradients. The dissipation flux is calculated as

$$
\vec{D}\left(Q_{i, j}\right)=d_{i+\frac{1}{2}, j}-d_{i-\frac{1}{2}, j}+d_{i, j+\frac{1}{2}}-d_{i, j-\frac{1}{2}}
$$

The terms on the right-hand side have a similar form and are a blend of first and third-order differences. For example,

$$
\begin{aligned}
d_{i+\frac{1}{2}, j}=\frac{\Omega_{i+\frac{1}{2}, j}}{\Delta t}\left\{\varepsilon_{i+\frac{1}{2}, j}^{(2)}\left(Q_{i+1, j}-Q_{i, j}\right)-\right. \\
\left.\varepsilon_{i+\frac{1}{2}, j}^{(4)}\left(Q_{i+2, j}-3 Q_{i+1, j}+3 Q_{i, j}-Q_{i-1, j}\right)\right\}
\end{aligned}
$$

Here, $\varepsilon^{(2)}$ and $\varepsilon^{(4)}$ are the coefficients of second- and fourthorder dissipation terms, respectively. The expression for these coefficients is given by

$$
\begin{aligned}
& \varepsilon_{i+\frac{1}{2}, j}^{(2)}=\gamma_{p}^{(2)} \max \left(v_{i+1, j}, v_{i, j}\right)+\gamma_{\rho}^{(2)} \max \left(\delta_{i+1, j}, \delta_{i, j}\right) \\
& \varepsilon_{i+\frac{1}{2}, j}^{(4)}=\max \left(0,\left(\gamma_{p}^{(4)}-\varepsilon_{i+\frac{1}{2}, j}^{(2)}\right)\right)
\end{aligned}
$$

where $v_{i, j}$ and $\delta_{i, j}$ are the sensors to activate the seconddifference numerical dissipation in the regions of strong gradients and to deactivate it elsewhere. The first term on the right-hand side of Eq. (20) is similar to that proposed initially by Jameson et al. [29]. $v_{i, j}$ is the pressure sensor and is applied in the regions with great pressure gradients, such as the shock and stagnation regions. The second term in Eq. (20) is a supplementary sensor added for cavitating flows [30]. Here, $\delta_{i, j}$ is the density sensor and is applied in the regions with noticeable density gradients, such as the cavity interface. These two sensors are defined as follows:

$$
\begin{gathered}
v_{i j}=\frac{\left|p_{i+1, j}-2 p_{i, j}+p_{i-1, j}\right|}{\left|p_{i+1, j}+2 p_{i, j}+p_{i-1, j}\right|} \\
\delta_{i j}=\frac{\left|\rho_{i+1, j}-2 \rho_{i, j}+\rho_{i-1, j}\right|}{\left|\rho_{i+1, j}+2 \rho_{i, j}+\rho_{i-1, j}\right|}
\end{gathered}
$$

The study indicates that the results of cavitating flows based on the solution of the multiphase Euler equations are sensitive to the numerical dissipation terms and these effects are investigated in the paper. 
Pseudo-temporal Discritization. An explicit multistage time-stepping method is used to discretize the pseudo-temporal term in Eq. (14). The solution is advanced in the pseudo-time $\tau$ using a four-stage Runge-Kutta scheme as

$Q_{i, j}^{0}=Q_{i, j}^{\tau}$

$Q_{i, j}^{n}=Q_{i, j}^{0}+\frac{\zeta_{k} \Delta \tau_{i, j}}{\Omega_{i, j}} R^{k-1}\left(\vec{Q}_{i, j}\right) \quad k=1,2,3,4$

$Q_{i, j}^{\tau+\Delta \tau}=Q_{i, j}^{4}$

where the parameters $\zeta_{k}$ are taken as $\frac{1}{4}, \frac{1}{3}, \frac{1}{2}$ and 1 . To accelerate the convergence rate of the solution to a steady state, the local time-stepping is employed. The local time step, $\Delta \tau_{i, j}$ is calculated as follows

$\Delta \tau_{i, j}=\frac{C F L \Delta \chi_{i, j}}{\left(\lambda_{\max }\right)_{i, j}}$

where $C F L$ is the Courant-Friedrichs-Lewy number and $\Delta \chi$ is the smallest face length of each cell. The parameter $\lambda_{\max }$ designates the maximum eigenvalue of the Jacobian matrix and is given by

$$
\lambda_{\max }=|\vec{V}|+\sqrt{|\vec{V}|^{2}+\beta^{2}}
$$

Boundary Conditions. The numerical solution of the system of differenced equations needs suitable boundary conditions to be implemented. The far field boundary conditions are applied at a finite distance from the body surface. The velocity components and volume fractions are specified at the inflow boundary and they are extrapolated at the outflow boundary, while the pressure is specified at the outflow boundary and it is extrapolated at the inflow boundary. Since the flow is assumed to be inviscid, the non-penetrating condition for the velocity components is enforced on the body surface. The boundary conditions on the body surface are imposed by using dummy cells with appropriate values at each pseudo-time step. The velocity components within a dummy cell, $(u, v)_{d}$, are evaluated from the values in the cell $(i, j)$ adjacent to the surface, $(u, v)_{i, j}$, as follows

$\left\{\begin{array}{l}u \\ v\end{array}\right\}_{d}=\left[\begin{array}{cc}1-2 \hat{n}_{x}^{2} & -2 \hat{n}_{x} \hat{n}_{y} \\ -2 \hat{n}_{x} \hat{n}_{y} & 1-2 \hat{n}_{y}^{2}\end{array}\right]\left\{\begin{array}{l}u \\ v\end{array}\right\}_{i, j}$

where $\hat{\boldsymbol{n}}\left(\hat{n}_{x}, \hat{n}_{y}\right)$ is the unit vector normal to the boundary face. The density, pressure and volume fraction within the dummy cell are set equal to the values in the cell adjacent to the surface.

\section{RESULTS AND DISCUSSION}

The multi-phase Euler system described in the present study is applied to compute the sheet- and super-cavitating flows over a variety of geometries. Since sheet and supercavitation have a quasi-steady behavior, with most of the unsteadiness localized in the rear closure region, steady state calculations can be regarded as a valid assumption in the simulations presented herein. The results are organized in three parts: The first simulation presented is the cavitating flow over the NACA 0012 hydrofoil for different conditions and the sensitivity of the solution to the numerical parameters such as the grid size and the dissipation coefficients are studied. The effects of the density ratio and the cavitation models on the results are also discussed. A comparison of the present solutions with the existing numerical results based on the interface tracking method [13] at different cavitation numbers and angleof-attacks is carried out in this section. Next, the computations are performed for the cavitating flow over the NACA 66 hydrofoil and the accuracy of the results is verified through comparing with the experimental measurements [31] and the numerical results [13]. In addition, the capability of the current algorithm to capture the mid-chord cavitation, kind of sheet cavitation where the cavitation inception occurs somewhere else other than the leading edge of the geometry, is demonstrated. Finally, a series of computation results is presented for the cavitating flow past an axisymmetric cylindrical headform. A sensitivity study is performed to examine the effects of the grid size and the dissipation parameters on the solution for this test case. The results for cavitating flow over this geometry are compared with existing experimental data of Rouse and McNown [32] and computations of Kunz et al. [20]. The liquid to gas density ratio is specified to be 100 and the Kunz et al. cavitation model is used in all the simulations unless otherwise indicated.

The NACA 0012 hydrofoil. The results of Deshpande et al. [13] are used as a baseline for comparing the results of cavitating flows since the inviscid cavitating flow has been computed there too. They used the Euler equations in conjunction with the interface tracking method. Herein, the computation of multiphase Euler equations using the interface capturing method is considered. A grid refinement study is performed to assess the grid resolution requirements around the NACA 0012 hydrofoil at 5 degree angle-of-attack and the cavitation number is set to be $\sigma=1$. A view of the hydrofoil and the computational domain near the body is shown in Figure 1 . Three grid systems, the coarse grid $(101 \times 51)$, the medium grid $(151 \times 75)$ and the fine grid $(201 \times 101)$; are used for this study.

As illustrated in Figure 2, the difference between the predicted surface pressures for the medium and fine meshes are small and the solution seems independent from the grid size when the grid points become larger than the medium one. Thus, the medium mesh system is utilized for the all subsequent calculations presented here because it costs less computation effort without affecting the accuracy of the solution. To examine the sensitivity of the solution to the dissipation 


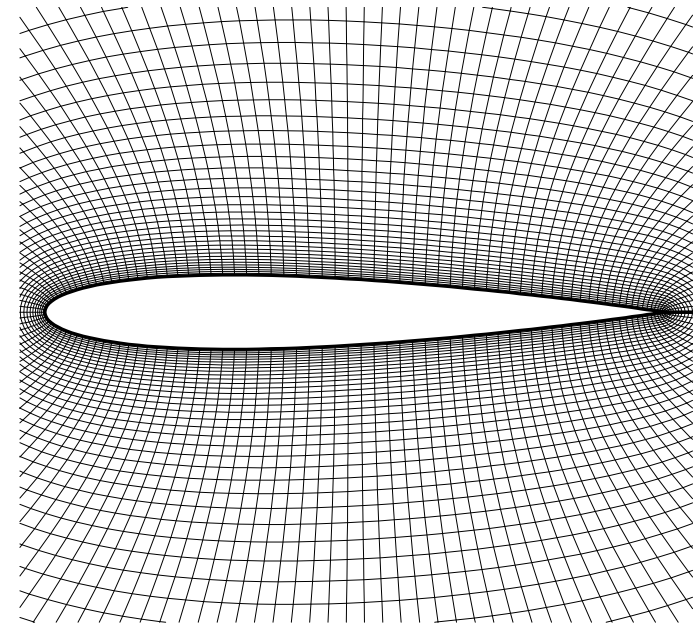

Fig. 1 Details of the NACA 0012 hydrofoil and the computational $O$-grid used in computation of cavitating flows.

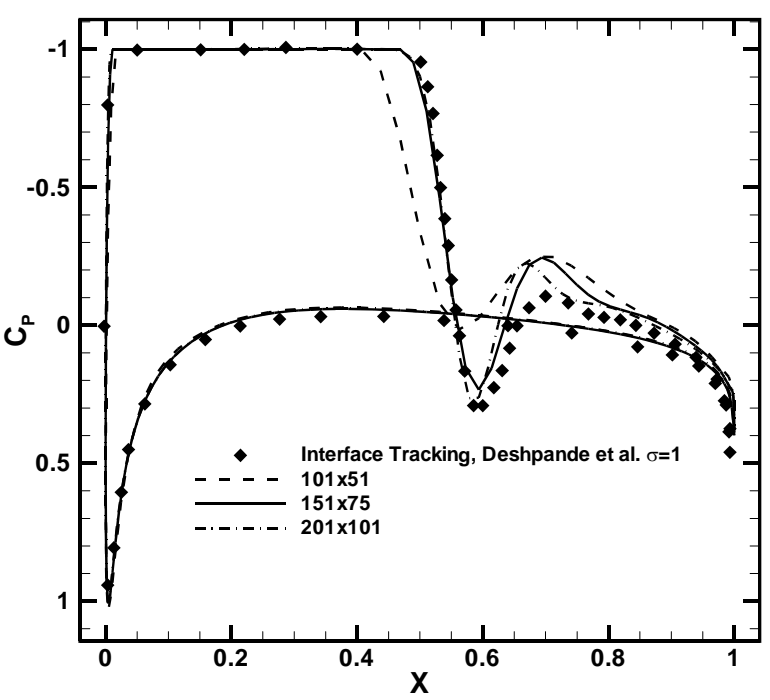

Fig. 2 Effect of grid refinement on surface pressure coefficient distribution for cavitating flow over the NACA 0012 hydrofoil at $\alpha=5^{0}$ and $\sigma=1$.

coefficients, the cavitating flow over the NACA 0012 at 5 degree angle-of-attack and $\sigma=1$ is considered again. Figure 3 illustrates the effects of the pressure sensor coefficient in the second-order term of the numerical dissipation, $k_{p}^{(2)}$, on the surface pressure coefficient distribution. The results indicate that the pressure sensor has no a stabilizing effect and even if this sensor in the second-order dissipation term is included then the solution is deteriorated near the cavity interface. Hence, this sensor is deactivated $\left(k_{p}^{(2)}=0\right)$ to obtain a more accurate solution.

Figures 4 and 5 demonstrate the surface pressure coefficient distribution and the convergence rate of the solution for the different values of the density sensor coefficients in the second- and fourth-order terms of the numerical dissipation. In contrast to the pressure sensor, the density sensor in the secondorder dissipation term has a significant effect on the solution

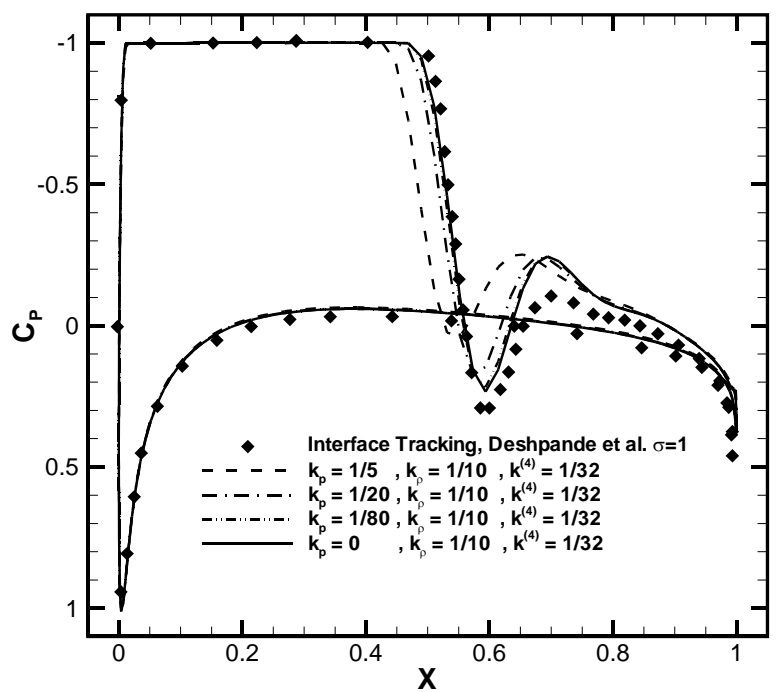

Fig. 3 Effect of $2^{\text {nd }}$-order dissipation coefficient based on pressure $k_{p}^{(2)}$ on surface pressure coefficient distribution for cavitating flow over the NACA0012 hydrofoil at $\alpha=5^{0}$ and $\sigma=1$.

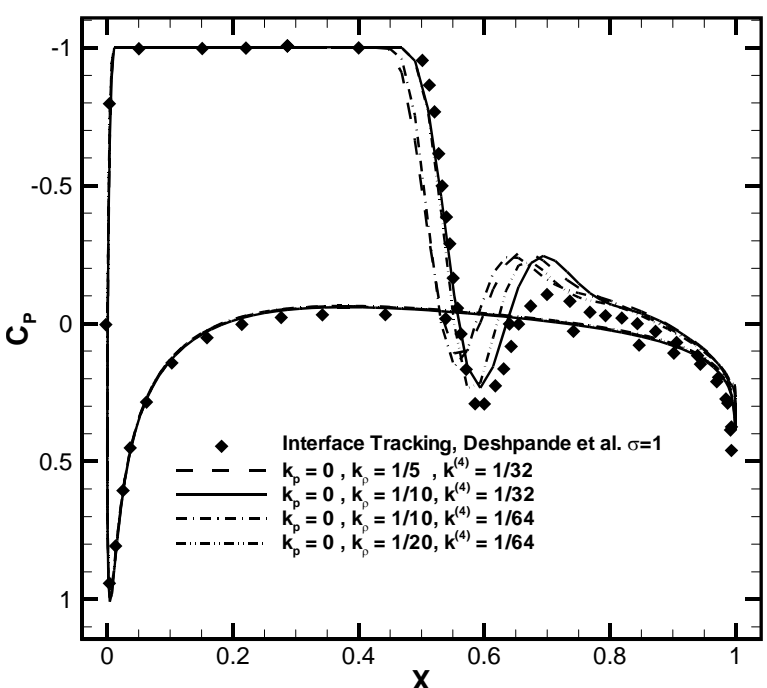

Fig. 4 Effect of $2^{\text {nd }}$ - and $4^{\text {th }}$-order dissipation coefficients on predicted surface pressure distribution for cavitating flow over the NACA 0012 hydrofoil at $\alpha=5^{0}$ and $\sigma=1$.

because of the strong density gradients at the cavity interface due to the phase change. Thus, the small values of this coefficient may lead to instabilities such that with a choice of $k_{\rho}^{(2)}=1 / 40$ the solution turned out to be quite unstable and led to divergence. On the other hand, larger values of density sensor coefficient cause an overdamping effect on the solution. Thus, a value of $k_{\rho}^{(2)}=1 / 10$ seems a suitable value for this coefficient. Since the $4^{\text {th }}$-order term has to suppress the tendency for odd and even point decoupling, setting the value of this coefficient to zero can cause severe oscillations which lead to instability and divergence of the solution. On the contrary, a higher value for this coefficient (i.e., $k^{(4)}=1 / 16$ ) results in the computation divergence. Choosing $k^{(4)}=1 / 32$ 


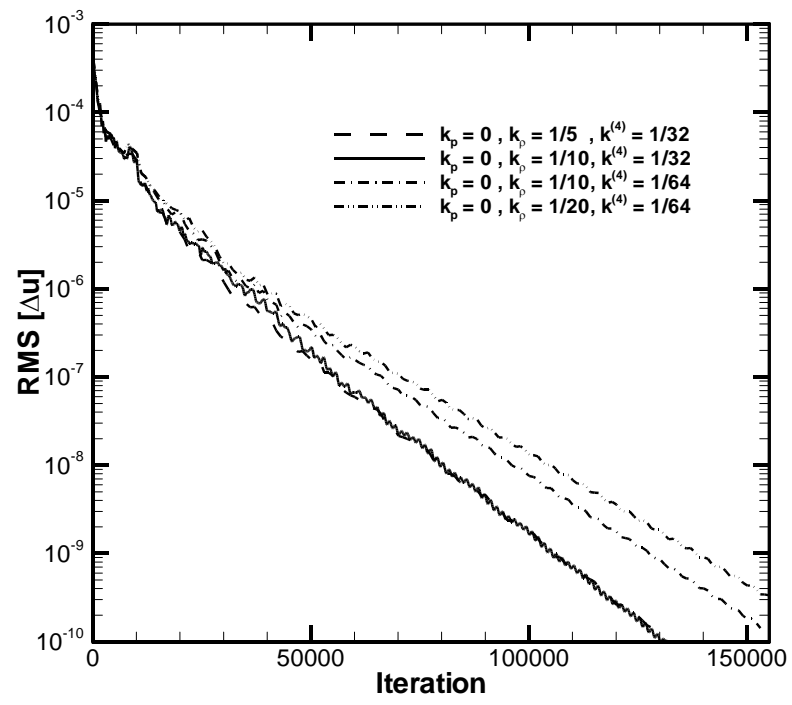

Fig. 5 Effect of $2^{\text {nd }}$ - and $4^{\text {th }}$-order dissipation coefficients on convergence rate of the solution for cavitating flow over the NACA 0012 hydrofoil at $\alpha=5$ and $\sigma=1$.

gives a stable and accurate solution enough. Note that the effect of dissipation terms is more pronounced at the aft end of the cavity region. It is clear that the $2^{\text {nd }}$-order and $4^{\text {th }}$-order dissipation terms have a reverse behavior there. While increasing the coefficient associated with the $2^{\text {nd }}$-order dissipation mostly shortens the cavity length, increasing the $4^{\text {th }}$ order dissipation lengthens it. The present study indicates that the $2^{\text {nd }}$-order and $4^{\text {th }}$-order dissipation values in the range of $k_{\rho}^{(2)}=1 / 20-1 / 10$ and $k^{(4)}=1 / 64-1 / 32$, are appropriate ones for an accurate modeling of cavitation. In Figures 6 and 7 , the surface pressure coefficient distribution and the convergence history are provided for the different density ratios for the simulation of cavitating flow over the NACA 0012 hydrofoil at 5 degree angle-of-attack and the cavitation number of $\sigma=1$. As the density ratio is increased, the surface pressure coefficient and the convergence history is changed somewhat, but beyond $\rho_{l} / \rho_{v}=10$, the performance of the solver is almost independent of the density ratio. This behavior retains the physically observed characteristic that the cavity size, and thereby the dynamics of the two-fluid motion, are nearly independent of liquid-vapor density ratio [20].

The sensitivity of the solution to two cavitation models is studied in Figure 8. It can be seen that using either Merkle et. al. or Kunz et. al. cavitation model has little effect on the surface pressure coefficient distribution predicted. The differences in the predictions are more pronounced at the closure region which is due to the different compressibility characteristics imposed by the cavitation models [2]. The study has shown using these two cavitation models, the convergence rates of the solution are nearly the same.

In Figure 9, the predicted surface pressure distribution for the cavitating flow over the NACA 0012 for the various cavitation numbers ( $\sigma=0.9,1.0$ and 1.2) at 5 degree angle-

of-attack are presented and compared with the results of interface tracking method of Deshpande et al. [13]. The computed cavity profiles are also plotted in this figure.

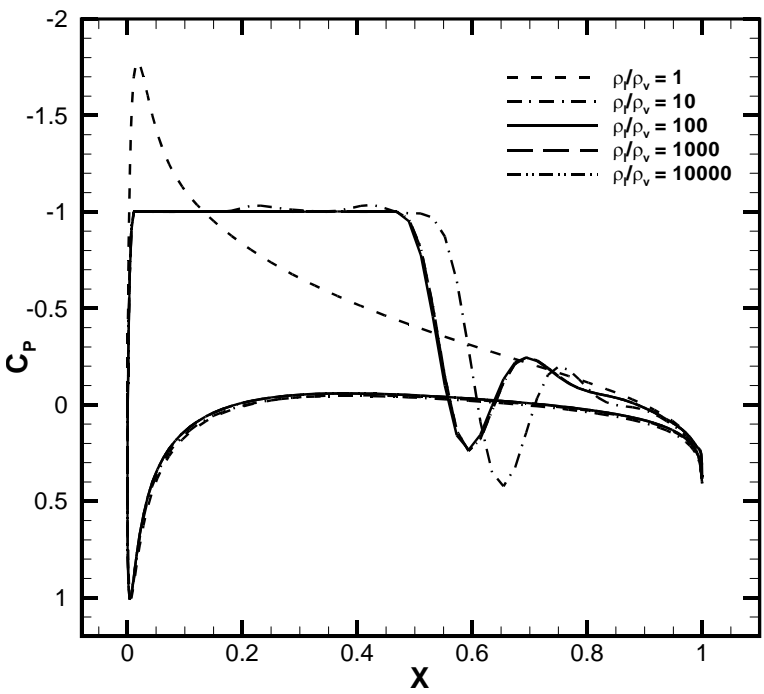

Fig. 6 Effect of density ratio $\rho_{l} / \rho_{v}$ on surface pressure coefficient distribution for cavitating flow over the NACA 0012 hydrofoil at $\alpha=5$ and $\sigma=1$.

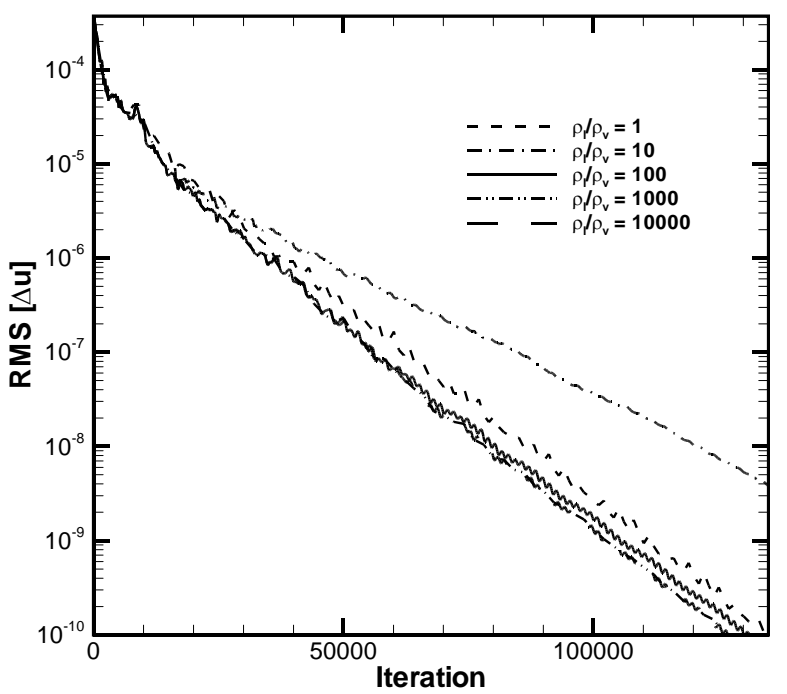

Fig. 7 Effect of density ratio $\rho_{l} / \rho_{v}$ on convergence rate of solution for cavitating flow over the NACA 0012 hydrofoil at $\alpha=5$ and $\sigma=1$.

A value of $\rho_{m}=0.95$ is adopted to show the cavity interface on all the subsequent plots. The present results based on the interface capturing method and those of Deshpande et al. based on the interface tracking method are very similar to each other despite in the aft end of the cavity region where a wake model has to be applied in an interface tracking approach. The good comparison between the two numerical results is an important validation of the present results which does not require a user specified wake closure model.

Figure 10 shows the surface density distribution on the airfoil surface relating to the same conditions. As seen from density plots, the liquid phase first expands and vapor phase appears uniformly inside the cavity, then the vapor phase compresses, in a shock like fashion, back to the liquid phase. 


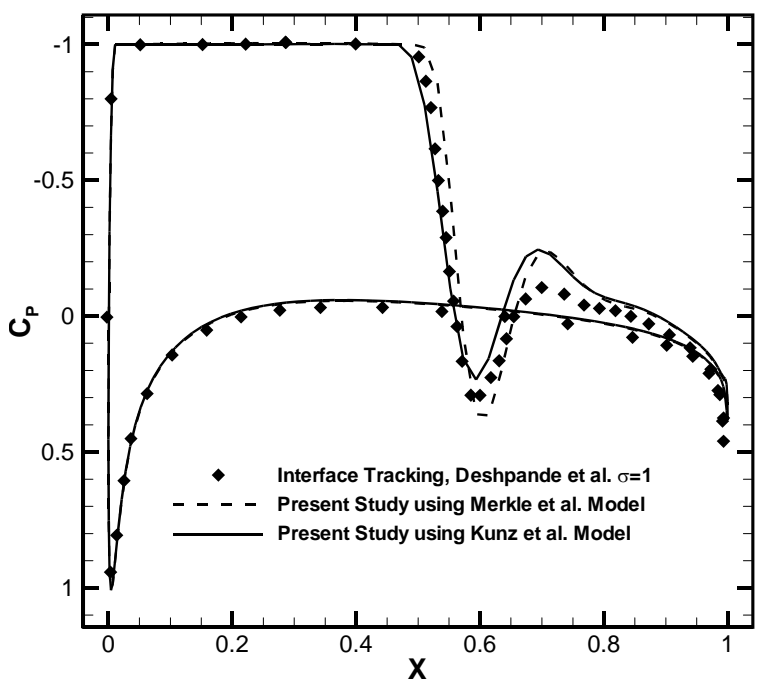

Fig. 8 Effect of cavitation models on surface pressure coefficient distribution for two cavitation models for cavitating flow over the NACA 0012 hydrofoil at $\alpha=5^{0}$ and $\sigma=1$.

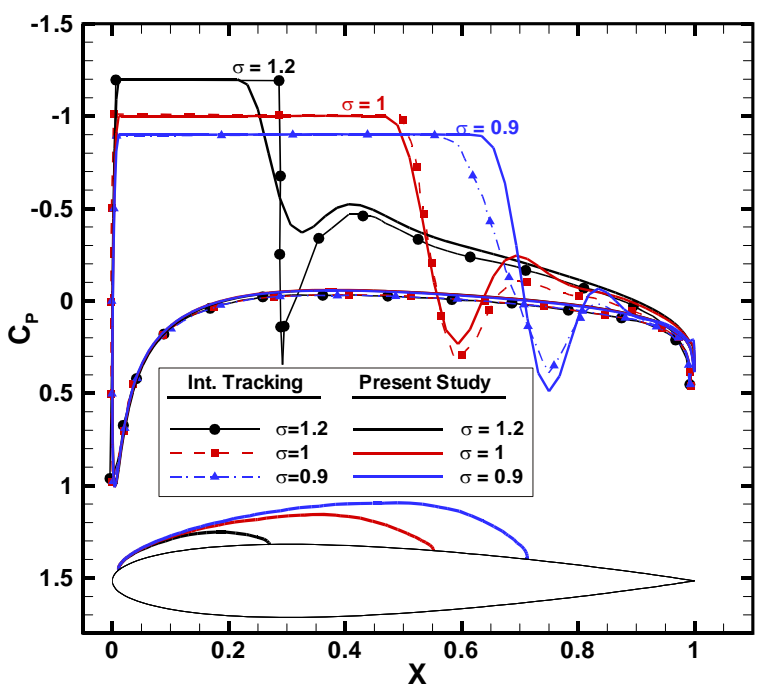

Fig. 9 Comparison of predicted surface pressure coefficient distribution with the results of interface tracking method for the

NACA 0012 hydrofoil at $\alpha=5^{0}$ and $\sigma=0.9,1.0,1.2$.

The differences among the three cavitation numbers imply that as the cavitation number is lowered the density distribution can better capture the actual density ratio that is provided.

In Figure 11, the surface pressure coefficient distribution is given for a fixed cavitation number $(\sigma=1.0)$ at three angles-of-attacks ( $\alpha=4^{\circ}, 5^{\circ}$ and $6^{\circ}$ ). The calculated cavity profiles are also depicted in this figure. The results indicate a growth in the cavity size with decreasing the cavitation number and increasing the angle-of-attack. Again the present computations based on the multiphase Euler equations using the interface capturing method are comparable with those of Deshpande et al. based on the Euler equations using the interface tracking method except in the aft end of the cavity region where a wake model has to be implemented in the interface tracking method to properly close the cavity region.

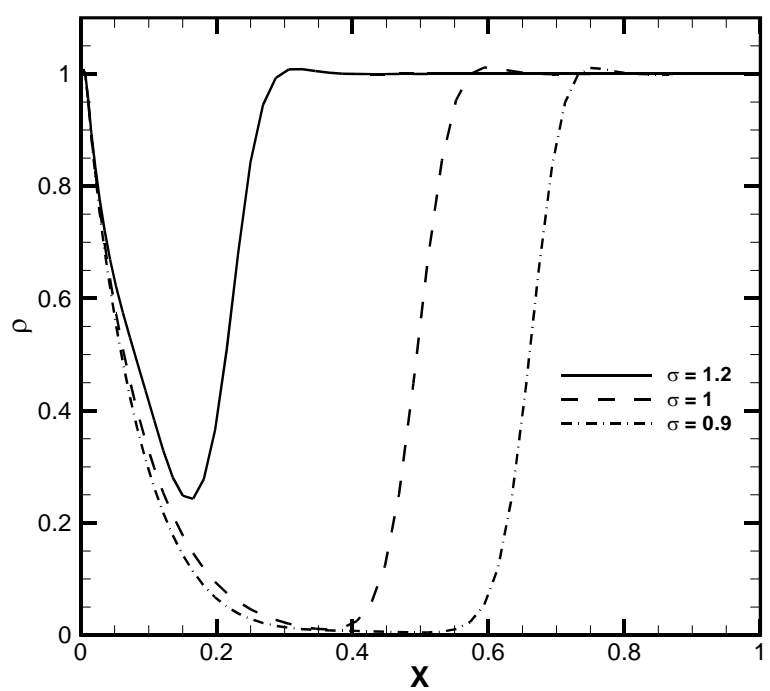

Fig. 10 Comparison of surface density distribution for the NACA 0012 hydrofoil at $\alpha=5^{0}$ and $\sigma=0.9,1.0,1.2$.

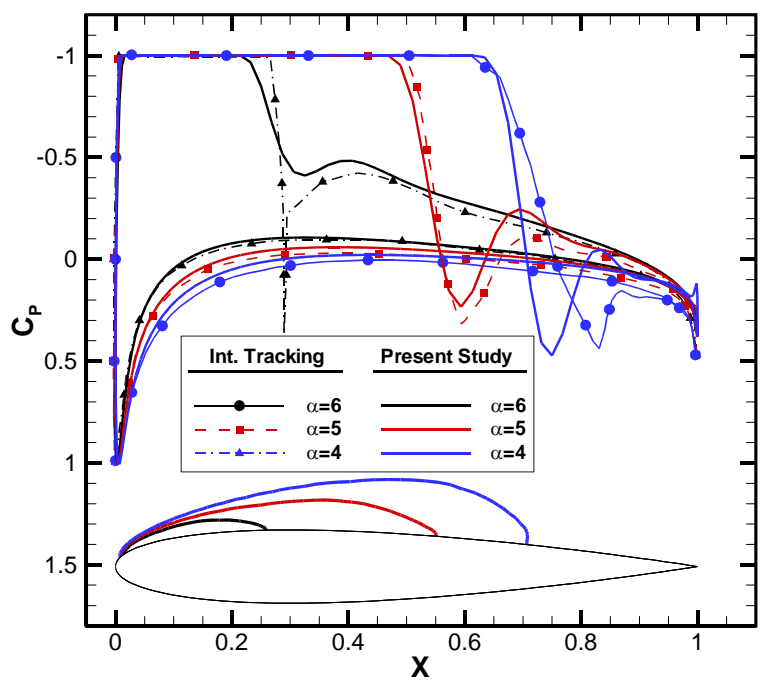

Fig. 11 Comparison of predicted surface pressure coefficient distribution with the results of interface tracking method for the NACA 0012 hydrofoil at $\sigma=1.0$ and $\alpha=4^{0}, 5^{0}, 6^{0}$ ).

Note that the most attractive feature of the present procedure is that no wake closure model is required and the whole cavity region shape is obtained as a part of the solution.

Figure 12 shows the density contours over the hydrofoil at a fixed angle-of-attack $\left(\alpha=5^{\circ}\right)$ and for the different cavitation numbers. It is obvious that the cavitation bubble volume increases as the cavitation number is decreased. Finally, the detailed predictions of supercavitation over this hydrofoil are illustrated in Figure 13. It can be seen that the cavitation bubble develops downstream in a way it envelopes the entire suction side of the hydrofoil. The results demonstrate that the present numerical treatment employing the multiphase Euler equations is capable of modeling of sheet and super-cavitating flows. 


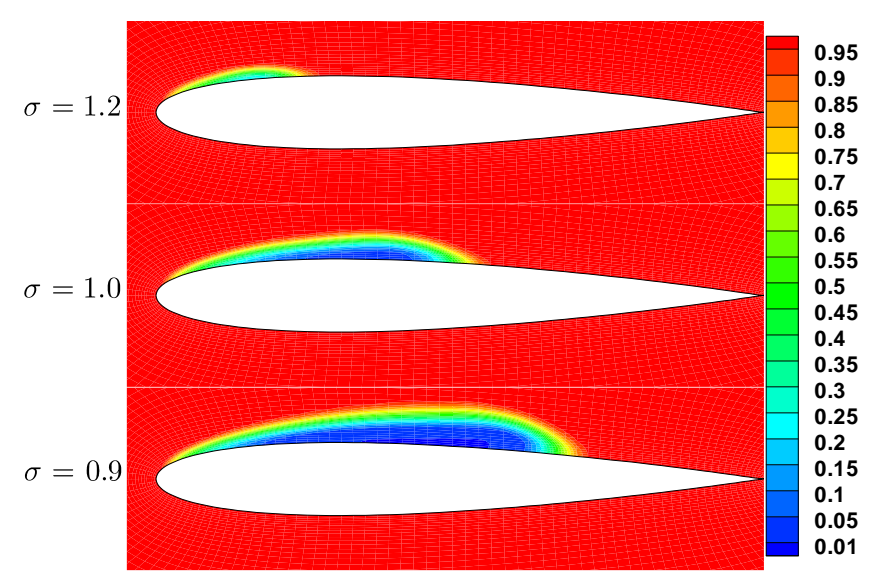

Fig. 12 Computed flow field shown by density contours for cavitating flow over the NACA 0012 hydrofoil at $\alpha=5^{0}$ and $\sigma=0.9,1.0,1.2$.

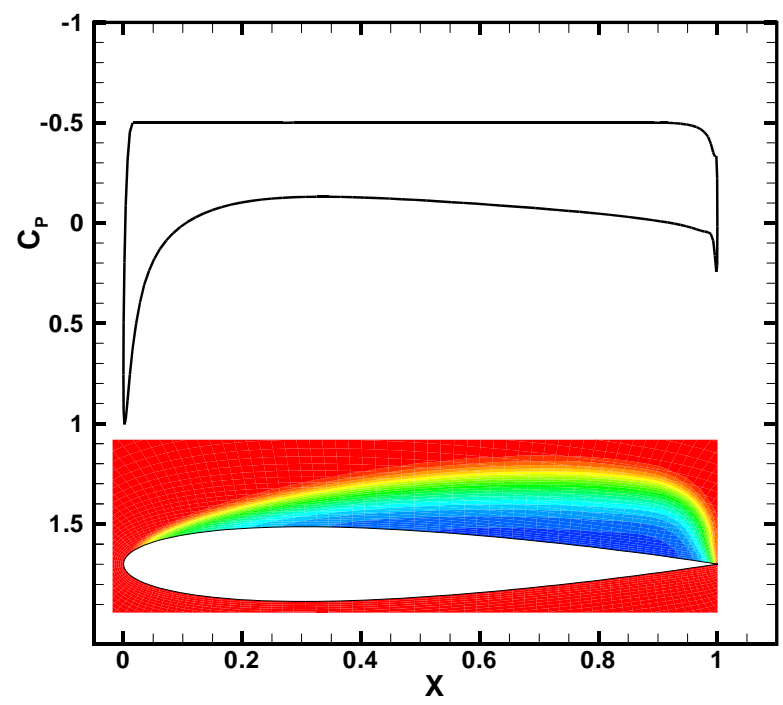

Fig. 13 Surface pressure distribution and computed density contours for supercavitating flow over the NACA 0012 hydrofoil at $\alpha=5^{0}$ and $\sigma=0.5$.

The NACA 66 hydrofoil. In this section, the results of the present computation of cavitating flow over the NACA 66 hydrofoil are presented. The computational mesh with $(181 \times 81)$ grid points is used for all the simulations. The hydrofoil geometry and the mesh used for the computations are shown in Figure 14.

The present predictions of the surface pressure distribution for this hydrofoil are compared with the experimental measurements by Shen and Dimotakis [31] and the numerical results by Deshpande et al. [13]. Figure 15 illustrates the pressure distribution for the cavitation inception at an angle-ofattack of 4 degree and the cavitation number of $\sigma=1.76$. In this case, a small cavitation bubble forms on the leading edge of the hydrofoil and with favoring the cavitation condition, the

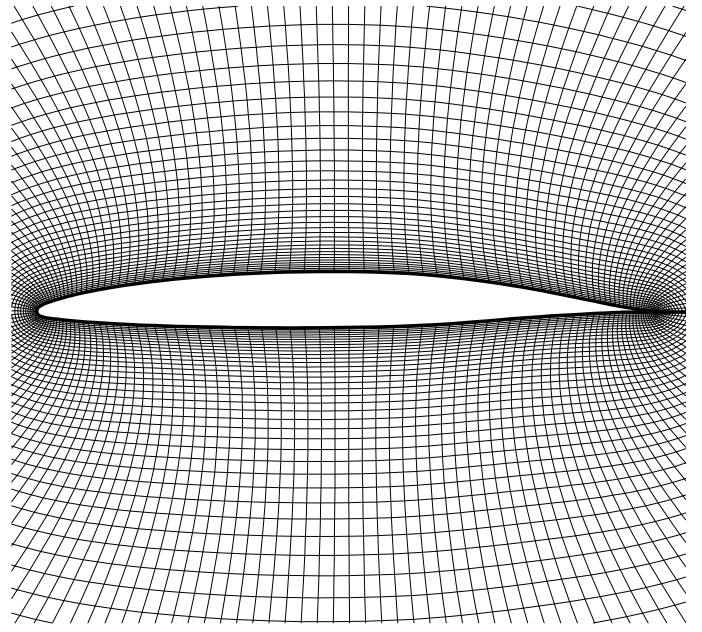

Fig. 14 Details of the NACA 66 hydrofoil and the computational Ogrid used in computation of cavitating flows.

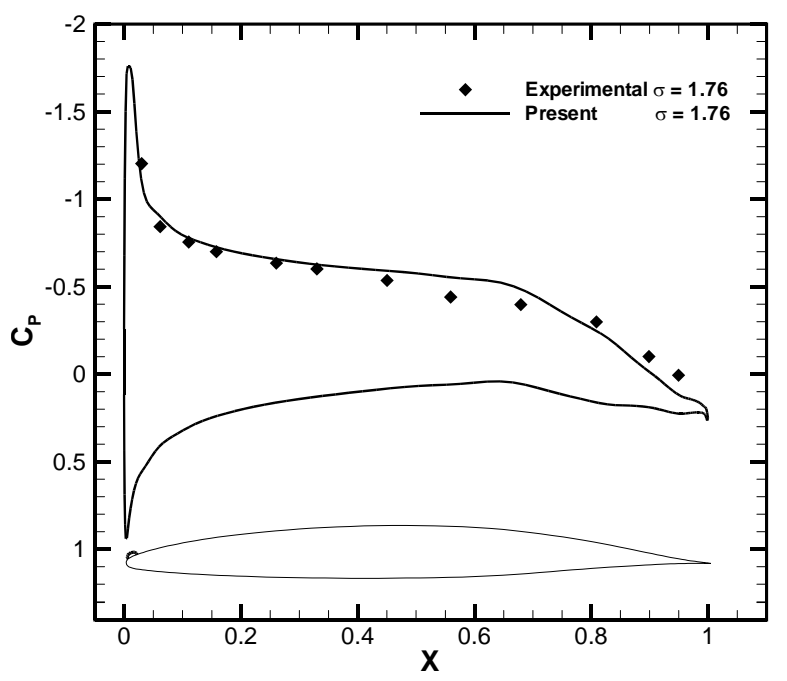

Fig. 15 Comparison of predicted pressure coefficient distribution with experimental data for leading-edge cavitation over the NACA 66 hydrofoil at $\alpha=4^{0}$ and $\sigma=1.76$.

cavity region is evolved. The surface pressure distribution compares very well with experimental data. Both the hydrofoil surface pressure distribution and the computed cavity profiles are shown in Figure 16. The results of cavitating flows for different cavitation numbers ( $\sigma=0.84,0.9$ and 1.0) are

carried out at a fixed angle-of-attack of 4 degree. The computed cavity profiles are also given in this figure. The study indicates the computed surface pressure distributions are in good agreement with the existing data. Again a slight discrepancy between the computed surface pressure distribution based on the interface capturing and that of the interface tracking [13], at the end of the cavity region, is because of using an ad-hoc wake closure model in the interface tracking approach at the end of the cavity region. However, a better agreement is observed between the current results and the experimental measurements. 

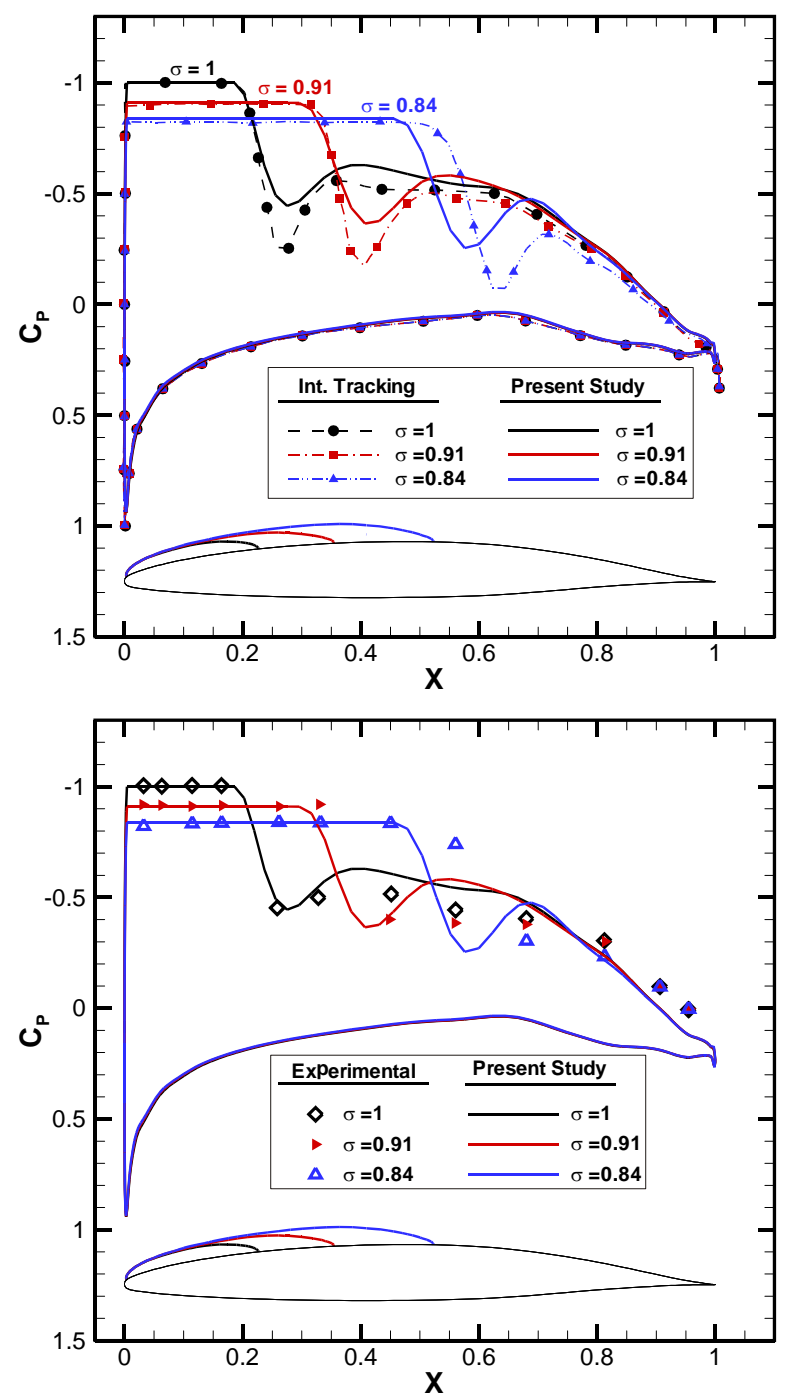

Fig. 16 Comparison of predicted pressure coefficient distribution with the results of interface tracking method and experimental data for cavitaing flow ove the NACA 66 hydrofoil at $\alpha=4^{0}$ and $\sigma=0.84,0.91,1.0$.

The weakness in capturing the aft end of the cavity region may be due to turbulence effects that can highly affect the structure of the wake region. Figure 17 depicts the density contours for the different cavitation numbers. The results show as the cavitation number is decreased, the cavity region grows.

The cavitating flow over the NACA 66 is computed at a small angle of attach $\left(\alpha=1^{\circ}\right)$ and the surface pressure distribution together with the cavity profile are presented in Figure 18. This condition is known as the mid-chord cavitation. The study shows that the computed surface pressure distribution exhibits good agreement with the experimental data. Since in the mid-chord cavitation, the location of the cavity inception is somewhere different than the leading edge of the hydrofoil, the results demonstrate the predictive capability of the present computations.

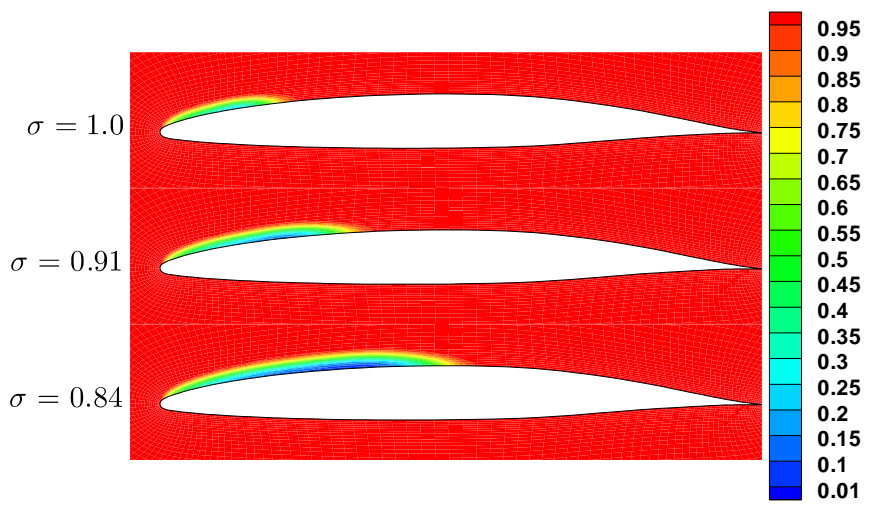

Fig. 17 Computed flow field shown by density contours for cavitating flow over the NACA 66 hydrofoil at $\alpha=4^{0}, \sigma=0.84,0.91,1.0$.

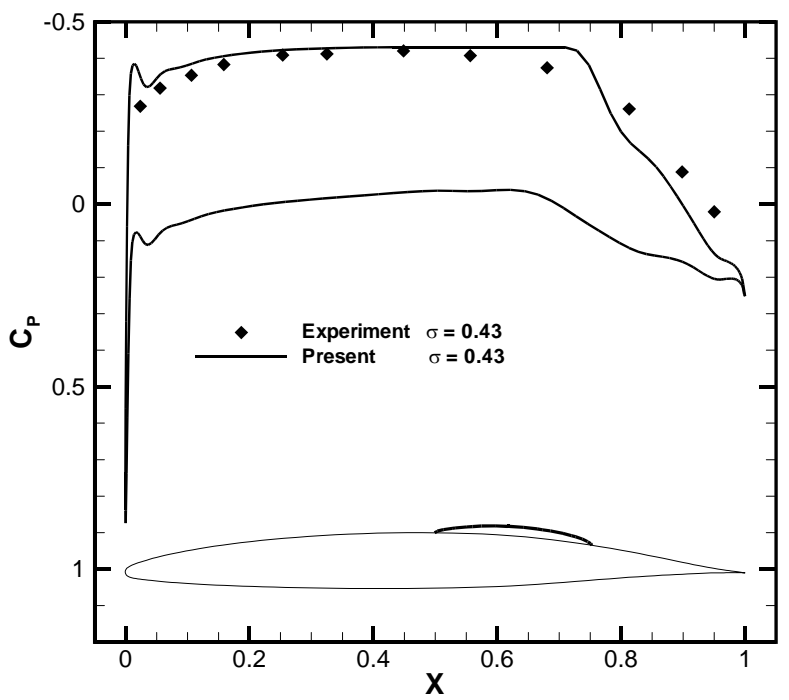

Fig. 18 Comparison of predicted pressure coefficient distribution with experimental data for mid-chord cavitation over the NACA 66 hydrofoil at $\alpha=1^{0}$ and $\sigma=0.43$.

The Cylindrical Headform. The last test case corresponds to the cavitating flow over a hemispherical headform as one of the most axisymmetric geometries that has been widely used for the assessment of numerical methods for modeling the cavitation. Rouse and McNown [32] have conducted experiments involving liquid water flow over this geometry. The present simulations are compared with the experimental data of Rouse and McNown, in which the surface pressure distribution for different cavitation numbers are reported. As in the NACA 0012, the grid independence study and sensitivity to the dissipation parameters are also discussed for this test case

A mesh dependency test is employed in order to pursue a solution independent of the grid size. An overview of the hemisphere headform and the near-field details of the computational grid used are shown in Figure 19. The results for the surface pressure distribution obtained with the three sets of 


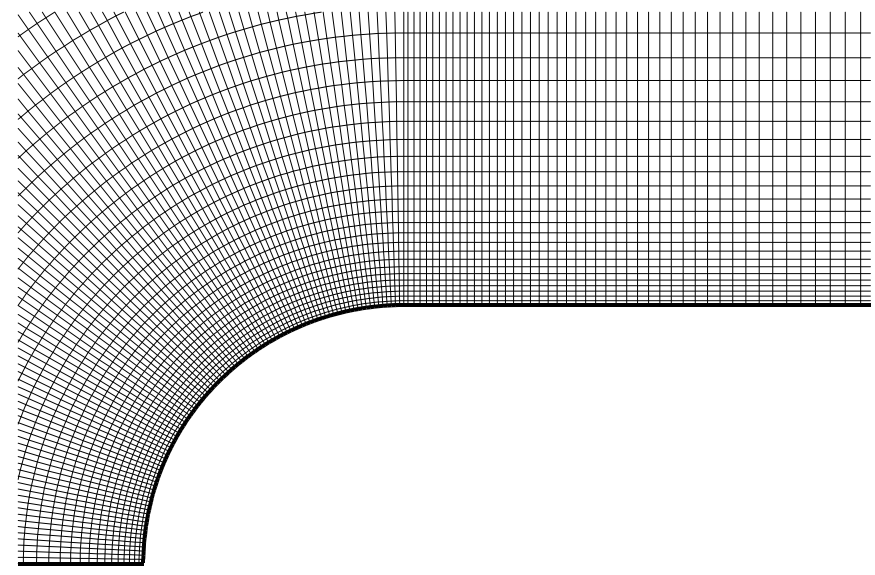

Fig. 19 Details of cylindrical headform geometry and the computational grid used in computation of cavitating flows.

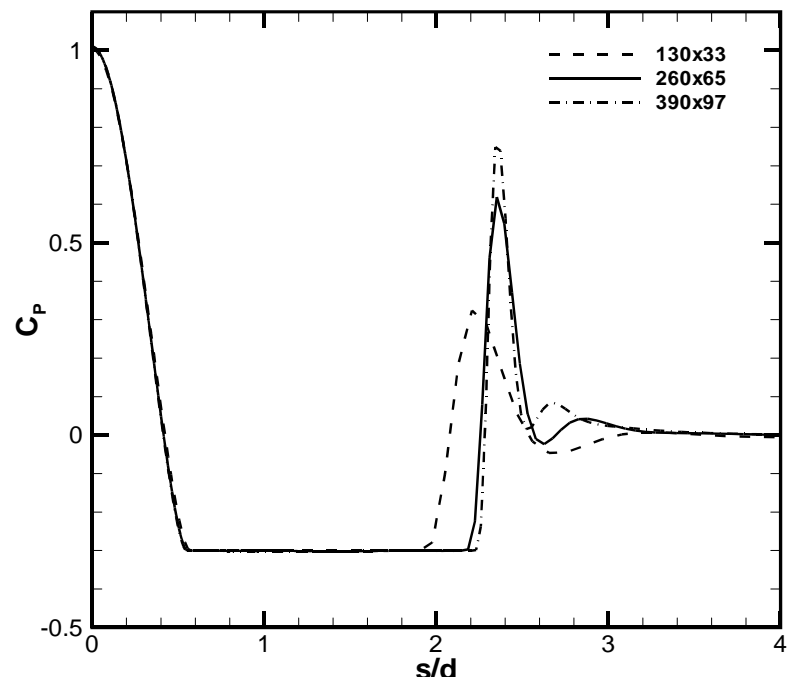

Fig. 20 Effect of grid refinement on surface pressure coefficient distribution for cavitating flow over the hemispherical headform at $\sigma=0.3$.

meshes, $(130 \times 33),(260 \times 65)$ and $(390 \times 97)$ are illustrated in Figure 20. The grid study is performed at a cavitation number of $\sigma=0.3$. It is obvious that the results obtained with the mesh sizes $(260 \times 65)$ and $(390 \times 97)$ are nearly the same and the results based on the mesh size $(130 \times 33)$ obviously deviate away from them. Thus, the medium mesh system $(260 \times 65)$ is employed for all subsequent calculations.

A sensitivity study of the solution to the dissipation coefficients at the same cavitation number $(\sigma=0.3)$ is performed. As discussed before for the NACA 0012 hydrofoil, the pressure sensor in the second-order dissipation term has no effect in stabilizing the solution and even the larger the value of its coefficient the more inaccurate the computed results. Thus, a value of $k_{p}^{(2)}=0$ is adopted for all the simulations that follow. The sensitivity of the computed surface pressure distribution to

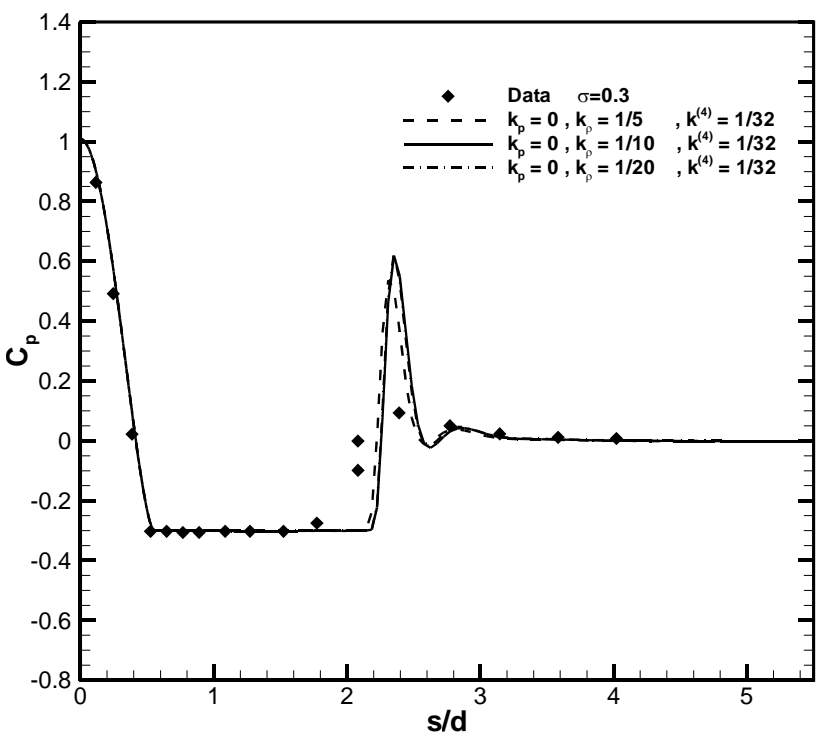

Fig. 21 Effect of $2^{\text {nd }}$-order dissipation coefficient based on density $k_{\rho}^{(2)}$ on surface pressure coefficient distribution for cavitating flow over the hemispherical headform at $\sigma=0.3$.

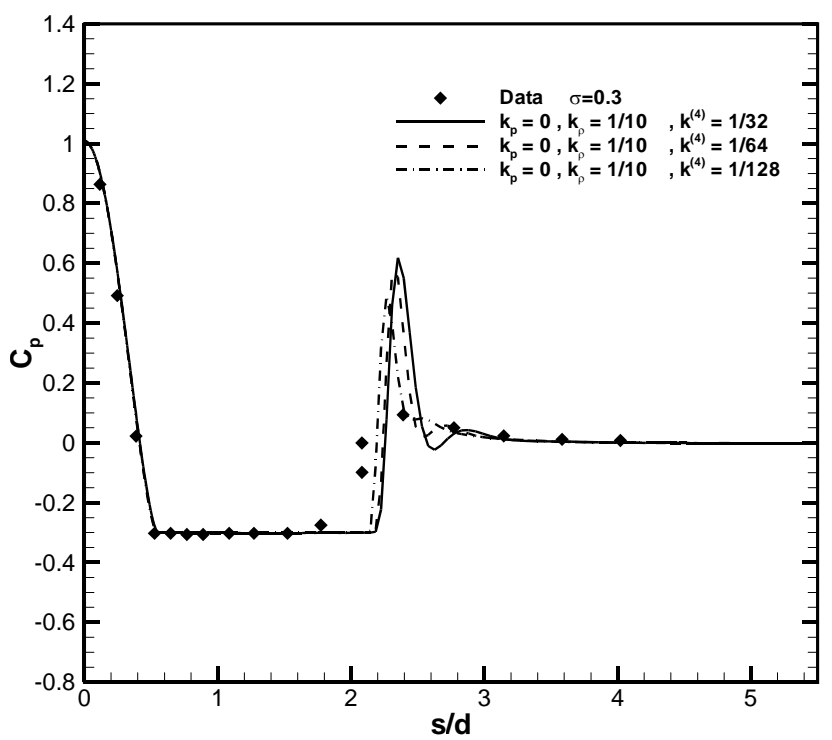

Fig. 22 Effect of $4^{\text {th }}$-order dissipation coefficient $k^{(4)}$ on surface pressure coefficient distribution for cavitating flow over the hemispherical headform at $\sigma=0.3$.

the density sensor coefficient, $k_{\rho}^{(2)}$ in the second-order dissipation term and to the coefficient of the fourth-order dissipation term $k^{(4)}$ and the results are given in Figures 21 and 22. It is obvious that the sensitivity study for this geometry indicates little effects to the dissipation coefficients compared to the hydrofoil case. This may be due to the combined effects of the geometry and the cavitation bubble. Figure 23 indicates the surface pressure distributions for the hemispherical headform at four cavitation numbers $(\sigma=0.2,0.3,0.4$ and 0.5 ) along with the noncavitating case. The present results for these conditions are compared with the experimental data [32] and the numerical results by Kunz et al. [20]. 

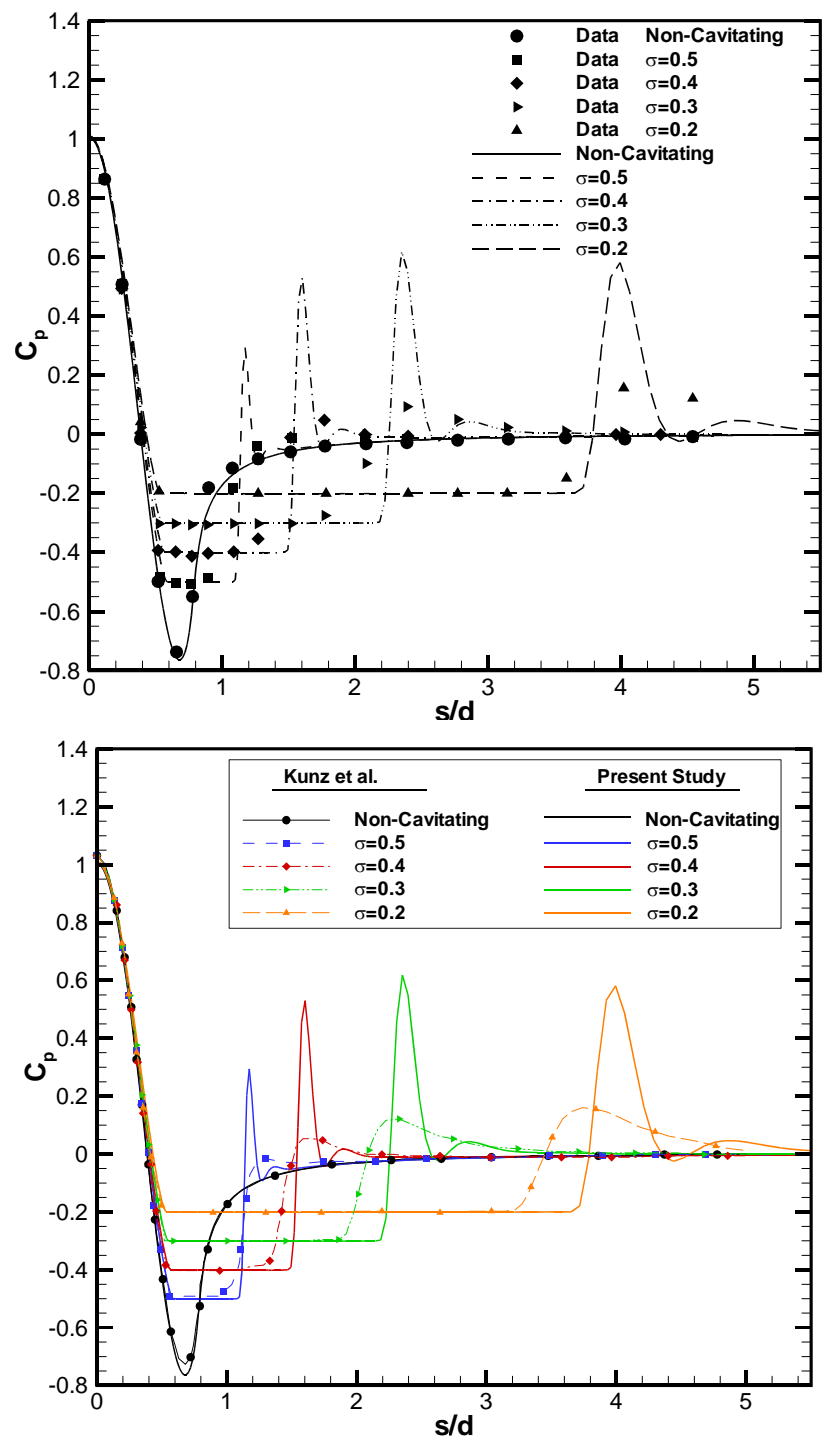

Fig. 23 Comparison of surface pressure coefficient distribution for cavitating flow over the hemispherical headform at

$$
\sigma=0.2,0.3,0.4,0.5 \text {. }
$$

The study shows the present numerical modeling performs well for both the noncavitating and cavitating conditions. The extent of the bubble is captured properly but the pressure recovery at the wake region of the cavity is too rapid which leads to a pressure overshoot compared to the experiments. This effect is most probably a consequence of the inviscid assumption, because the wake region is strongly influenced by the thermodynamic and turbulence effects. Figure 24 shows the density contours over this geometry for the cavitation numbers mentioned above. It is clear that the cavitation bubble volume increases as the cavitation number is decreased.

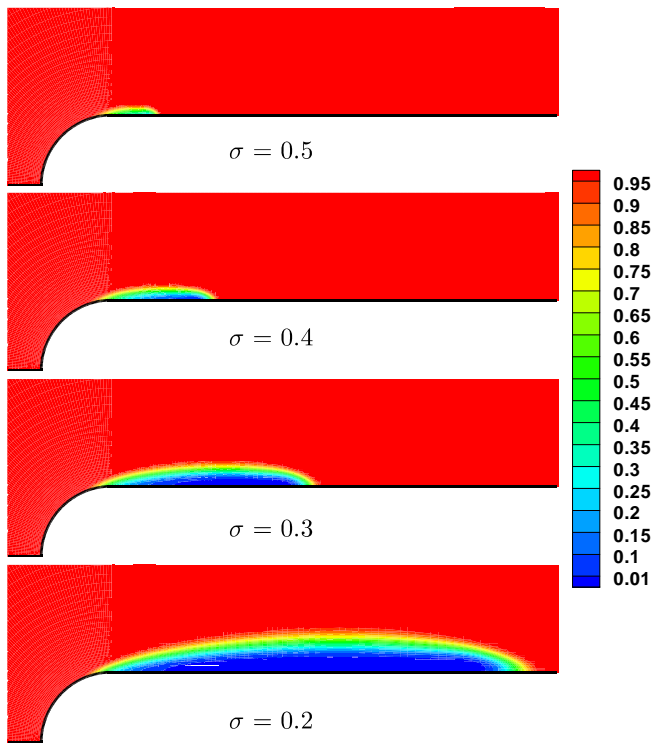

Fig. 24 Computed flow field shown by density contours for cavitating flow over the hemispherical headform at $\sigma=0.2,0.3,0.4,0.5$.

\section{CONCLUSIONS}

A numerical treatment for the modeling of cavitating flows is presented and assessed. The algorithm uses the preconditioned, homogenous, multiphase Euler equations with appropriate mass transfer terms. A cell-centered finite-volume scheme employing suitable dissipation terms to account for density jumps across the cavity interface is used to solve the multiphase Euler equations. The computations are presented for cavitating flows around the NACA0012 and NACA66(MOD) hydrofoils and also an axisymmetric hemispherical fore-body for different conditions. The study shows that some certain characteristics of cavitating flows can be obtained using the solution of the multiphase Euler equations that require low computational efforts compared to the multiphase NavierStokes equations. Owing the sensitivity of the system of Euler equations to the numerical instabilities, a sensitivity study is conducted to evaluate the effects of various parameters such as numerical dissipation coefficients and grid size on the accuracy and performance of the solution. The computations indicate that the results of cavitating flows based on the Euler equations are sensitive to the grid size and especially the numerical dissipation terms used for stabilizing the numerical algorithm. The present results are compared with the available numerical and experimental data which show good agreement. The present solution procedure based on the preconditioned multiphase Euler equations provides a good engineering prediction for different types of cavitating flows over 2D/axisymmetric geometries.

\section{ACKNOWLEDGEMENTS}

The authors would like to thank Sharif University of Technology for financial support of this research. 


\section{NOMENCLATURE}

\begin{tabular}{|c|c|}
\hline$u, v$ & $=$ Cartesian velocity components \\
\hline$p$ & $=$ pressure \\
\hline$E, F, G$ & $=$ inviscid flux vectors \\
\hline$S$ & $=$ source vector \\
\hline$\dot{m}$ & $=$ mass transfer rate \\
\hline$D$ & $=$ numerical dissipation vector \\
\hline$x, y$ & $=$ Cartesian coordinates \\
\hline$C_{\text {dest }}, C_{\text {prod }}$ & $=$ cavitation model constants \\
\hline$Q$ & $=$ flow-field vector, $Q=\left(p, u, v, \alpha_{l}\right)^{T}$ \\
\hline$C_{p}$ & $=$ pressure coefficient \\
\hline$t, t_{\infty}$ & $=$ time coordinate, mean flow time scale $\left.\frac{L}{U_{\infty}}\right)$ \\
\hline$\alpha$ & $=$ volume fraction or angle of attack \\
\hline$\beta$ & $=$ preconditioning parameter \\
\hline$\Gamma$ & $=$ preconditioning matrix \\
\hline$\lambda$ & $=$ eigenvalue \\
\hline$\rho$ & $=$ density \\
\hline$\sigma$ & $=$ cavitation number \\
\hline$\nu$ & $=$ pressure dissipation sensor \\
\hline$\delta$ & $=$ density dissipation sensor \\
\hline$\tau$ & $=$ pseudo-time coordinate \\
\hline $\begin{array}{l}\text { Subscript } \\
\text { Superscript }\end{array}$ & / \\
\hline$i, j$ & $=$ coordinate indices \\
\hline$l$ & $=$ liquid \\
\hline$m$ & $=$ mixture \\
\hline$v$ & $=$ vapor \\
\hline$\infty$ & $=$ free stream \\
\hline$+1-$ & $=$ production/destruction \\
\hline
\end{tabular}




\section{REFERENCES}

[1] Lecoffre, Y., 1999, Cavitation: Bubble Trackers, AA Balkema, Rotterdam.

[2] Senocak, I., 2002, "Computational Methodology for the Simulation of Turbulent Cavitating Flows," Ph.D. thesis, Florida State University.

[3] Gopalan, S., and Katz, J., 2000, "Flow Structure and Modeling Issues in the Closure Region of Attached Cavitation," Phys. of Fluids, 12(4), pp. 186-192.

[4] Li, J., Liu, L., and Feng, Z., 2007, "Numerical Prediction of the Hydrodynamic Performance of a Centrifugal Pump in Cavitating Flows," Commun. Numer. Meth. Engng, 23 pp. 363-384.

[5] Brennen, B., 1969, "A Numerical Solution of Axisymmetric Cavity Flows," J. Fluid Mech., 37 pp. 671-688.

[6] Furness, R. A., and Hutton, S. P., 1975, "Experimental and Theoritical Studies of Two Dimensional Fixed Type Cavities," J. Fluids Eng., 97 pp. 515-522.

[7] Guerst, J. A., 1959, "Linearized Theory for Partially Cavitating Hydrofoils."

[8] Kinnas, S., and Fine, N., 1993, "A Numerical Nonlinear Analysis of the Fluid around Two- and Three- Dimensinal Partially Cavitating Hydrofoils," J. Fluid Mech., 254 pp. 151-181.

[9] Kinnas, S. A., Sun, H., and Lee, H., 2003, "Numerical Analysis of Flow around the Cavitating Cav2003 Hydrofoil," Proc. Fifth International Symposium on Cavitation, Osaka, Japan.

[10] Lee, C. S., Kim, Y. G., and Lee, J. T., 1992, "A PontentialBased Panel Method for the Analysis of a Two Dimesnsional Super- or Partially-Cavitating Hydrofoils " J. Ship Research, 36 pp. 168-181.

[11] Ulhman, J. S., 1987, "The Surface Singularity Method Applied to Partially Cavitating Hydrofoils," J. Ship Research, 31(2).

[12] Chen, Y., and Heister, S. D., 1994, "A Numerical Treatment for Attached Cavitation," ASME J. Fluids Eng., 116 pp. 613-618.

[13] Deshpande, M., Feng, J., and Merkle, C. L., 1994, "Cavity Flow Predictions Based on the Euler Equations," ASME J. Fluids Eng., 116, 36-44.

[14] Qin, Q., Song, C. C. S., and Arndt, R. E. A., 2003, "A Virtual Single-Phse Natural Cavitation Model and Its Application to Cav2003 Hydrofoil," Proc. Fifth International Symposium on Cavitation (Cav2003), Osaka, Japan.

[15] Grogger, H. A., and Alajbegovic, A., 1998, "Calculation of Cavitating Flows in Venturi Geometries Using Two Fluid Model," Proc. ASME FEDSM 98, Washington D.C.

[16] Coutier-Delgosha, O., Deniset, F., Astolfi, J. A., and Leroux, J.-B., 2007, "Numerical Prediction of Cavitating Flow on a Two-Dimensional Symmetrical Hydrofoil and Comparison to Experiments," J. Fluids Eng., 129 pp. 279-292.

[17] Delannoy, Y., and Kueny, J. L., 1990, "Two Phase Flow Approach in Unsteady Cavitation Modeling," ASME-FED.
[18] Song, C. C. S., and Hi, J., 1998, "Numerical Simulations of Cavitating Flows with a Single-Phase Approach," Proc. Third International Symposium on Cavitation, Grenoble, France.

[19] Ventikos, Y., and Tzabiras, G., 2000, "A Numerical Method for the Simulation of Steady and Unsteady Cavitating Flows," Computers and Fluids, 29 pp. 63-88.

[20] Kunz, R.F., Lindau, J.W., Billet, M.L., Stinebring, D.R. (2001) "Multiphase CFD Modeling of Developed and Supercavitating Flows," VKI Special Course on Supercavitating Flows, February.

[21] Merkle, C. L., Feng, J. Z., and Buelow, P. E., 1998, "Computational Modeling of the Dynamics of Sheet Cavitation," Proc. 3rd International Symposium on Cavitation, J. M. Michel, et al., eds., Grenoble, France, 2, pp. 307-314.

[22] Chen, Y., and Heister, S. D., 1996, "Modeling Hydrodynamic Nonequilibrium in Cavitating Flows," ASME J. Fluids Eng., 118 pp. 172-178.

[23] Kubota, A., Kato, H., and Yamaguchi, H., 1992, "A New Modelling of Cavitating Flows : A Numerical Study of Unsteady Cavitation on a Hydrofoil Section," J. Fluid Mech., 240 pp. 59-96.

[24] Singhal, A. K., Li, H. Y., Athavale, M. M., and Jiang, Y., 2001, "Mathematical Basis and Validation of the Full Cavitation Model," New Orleans, Louisiana.

[25] Senocak, I., and Shyy, W., 2002, "A Pressure-Based Method for Turbulent Cavitating Flow Computations," J. of Computational Physics, 176 pp. 363-383.

[26] Wu, J., Utturkar, Y., and Shyy, W., 2003, "Assessment of Modeling Strategies for Cavitating Flow around a Hydrofoil," Proc. Fifth International Symposium on Cavitation (Cav2003), Osaka, Japan.

[27] Ahuja, V., Hosangadi, A., and Arunajatesan, S., 2001, "Simulations of Cavitating Flows Using Hybrid Unstructured Meshes," ASME J. Fluids Eng., 123 pp. 331340.

[28] Chorin, A. J., 1967, "A Numerical Method for Solving Incompressible Viscous Flow Problems," J. of Computational Physics, 2 pp. 12-26.

[29] Jameson, A., Schmidt, W., and Turkel, E., 1981, "Numerical Solutions of the Euler Equations by Finite Volume Methods Using Runge-Kutta Time-Stepping Schemes," AIAA paper 81-1259.

[30] Coutier-Delgosha, O., Fortes-Patella, R., Reboud, J. L., Hakimi, N., and Hirsch, C., 2005, "Stability of Preconditioned Navier-Stokes Equations Associated with a Cavitation Model," Computers and Fluids, 34 pp. 319-349.

[31] Shen, Y. J., and Dimotakis, P. E., 1989, "The Influence of Surface Cavitation on Hydrodynamic Forces," Proc. 22nd ATTC, St. Johns.

[32] Rouse, H., and Mcnown, J. S., 1948, "Cavitation and Pressure Distribution, Head Forms at Zero Angle of Yaw," State University of Iowa. 